UDC 535:530.182

\title{
Hyperbolic metamaterials based on graphene sheets with different values of chemical potentials
}

\author{
Zh.A. Kudyshev ${ }^{1}$, I.R. Gabitov ${ }^{2}$, A. Kissan ${ }^{3}$, Ye.S. Mukhametkarimov ${ }^{3 *}$ \\ ${ }^{l}$ Purdue University, Birck Nanotechnology Center, 610 Purdue Mall, IN 47907, West Lafayette, USA \\ ${ }^{2}$ Department of Mathematics, University of Arizona, 617 N. Santa Rita Ave, AZ 85721,Tucson, USA \\ ${ }^{3}$ National Nanotechnological Laboratory of Open Type, \\ Al-Farabi Kazakh National University, 71, Al-Farabi Ave., 050040, Almaty, Kazakhstan \\ *e-mail: yerzhan.mukhametkarimov@kaznu.kz
}

\begin{abstract}
In this paper possibility of creating a medium based on graphene-dielectric structures with the hyperbolic shape wave number dispersion is considered for the case when two types of graphene sheets with different chemical potentials periodically alternate. Using the Bloch theorem and the transfer matrix method, dispersion relations for the light wave numbers are derived for the layered structure and the possibility is demonstrated to manipulate the light propagation. The optical conductivity of graphene sheets is known to be frequency dependent and in the present scrutiny it is estimated via Kubo formula which simultaneously accounts for both the inter band and the intraband transitions of electrons. To apply the transfer matrix method we routinely express the amplitudes of the incident and reflected radiation at the input to the layered structure in terms of the amplitudes of electromagnetic waves at the output. The transmission spectrum of the layered dielectric medium which is sliced by two types of graphene sheets with different chemical potentials have a staircase behavior. It is demonstrated that the jump points in the transmission spectrum are simply controlled by adjusting the values of chemical potentials of graphene sheets.
\end{abstract}

Key words: hyperbolic metamaterials, graphene, dispersion of electromagnetic waves, transmission, absorption PACS number(s): 78.20.-e; 78.20.Bh; 78.20.Ci;

DOI: by publisher

\section{Introduction}

With the rapid development of nanotechnology it has become possible to create entirely new materials with a variety of unusual physical properties. These certainly include photonic crystals [1], metamaterials [2,3], and a number of other man-made materials. Among them a special place is occupied by the so-called hyperbolic materials, i.e. nano composites fabricated by the creation of a periodic structure of alternating layers of metal and dielectric media or by deposition of metal nanorods on a dielectric substrate [4]. A distinctive feature of nanocomposite materials is that their dielectric constant is described by the diagonal tensor whose components have different signs in a certain range of frequencies. In this case, it is conventional to speak of the hyperbolic "meta" material, in which, for instance, a phenomenon of negative light refraction is usually observed. Functionality of hyperbolic metamaterials is vastly confirmed by a large number of publications on the observation of various optical effects. In particular, it was demonstrated that metamaterials can help in overcoming the diffraction limit, in creating optical resonators and biosensors, as well as in observing nonlinear effects at rather low intensity of electromagnetic fields $[4,5]$.

Graphene, due to its unique optical properties, is a more promising material for creating hyperbolic metamaterials than conventional metals. This statement is based on the fact that it is possible to control the light transmission through a layered structure by changing its chemical potential [7]. Electrodynamic properties of a graphene, a two-dimensional lattice of carbon atoms, are characterized by its surface conductivity, which can be phenomenologically defined within the Kubo formalism [7-8].

\section{Surface conductivity and permittivity of a graphene}

Graphene is the first known truly twodimensional crystal. Unlike earlier attempts to create two-dimensional conductive layers, for example, two-dimensional electron gas, from semiconductors by controlling the width of the 
forbidden band, electrons in graphene are much more localized in the plane.

The variety of chemical and physical properties is due to the crystal structure and $\pi$-electrons of the carbon atoms that make up graphene. Wide study of the material in universities and research laboratories is primarily due to its availability and ease of its preparation using mechanical splitting of graphite crystals. The material, which showed its unique properties like high electrical and thermal conductivities, rather high mechanical strength etc., is interesting not only for researches, but also for engineers.
The motion of free electrons in graphene can be described using the Dirac equation, since charge carriers in graphene have a linear dispersion law near the Dirac points [9].In the absence of an external static magnetic field and under neglect of the spatial dispersion, the surface conductivity is determined as a scalar function of frequency $\omega$, the chemical potential $\mu$ (which depends on a voltage applied and/or on the chemical doping), phenomenological scattering rate $\Gamma$ (or the corresponding relaxation time $\tau=1 /(2 \Gamma)$ ), and the temperature $T$ :

$$
\sigma_{g}\left(\omega, \mu_{c}, \Gamma, \mathrm{T}\right)=-\frac{i e^{2}(\omega+i 2 \Gamma)}{\pi \hbar^{2}}\left[\frac{1}{(\omega+i 2 \Gamma)^{2}} \int_{0}^{\infty} \varepsilon\left(\frac{\partial f_{d}(\varepsilon)}{\partial \varepsilon}-\frac{\partial f_{d}(-\varepsilon)}{\partial \varepsilon}\right) d \varepsilon-\int_{0}^{\infty}\left(\frac{f_{d}(-\varepsilon)-f_{d}(\varepsilon)}{(\omega+i 2 \Gamma)^{2}-4(\varepsilon / \hbar)^{2}}\right) d \varepsilon\right]
$$

Here $\varepsilon$ is the energy, $\hbar$ denotes the Planck's constant, $e$ designates the elementary charge, $k_{B}$ stands for the Boltzmann constant, and $f_{d}(\varepsilon)=\left(\exp \left(\varepsilon-\left|\mu_{c}\right| / k_{B} T\right)+1\right)^{-1}$ is the distribution function in the Fermi-Dirac statistics.
The surface conductivity $\sigma_{g}\left(\omega, \mu_{c}, \Gamma, \mathrm{T}\right)$ of graphene consists of two main contributions, namely the intraband $\sigma_{\text {intra }}$ and the interband $\sigma_{\text {inter }}$ conductivities, respectively. The former can be written in the following analytic form:

$$
\sigma_{\text {intra }}\left(\omega, \mu_{c}, \Gamma, \mathrm{T}\right)=i \frac{e^{2} k_{B} T}{\pi \hbar^{2}(i 2 \Gamma+\omega)}\left[\frac{\mu_{c}}{k_{B} T}+2 \ln \left(\exp \left(-\frac{\mu_{c}}{k_{B} T}\right)+1\right)\right],
$$

whereas the second one reads as

$$
\sigma_{\text {inter }}\left(\omega, \mu_{c}, \Gamma, \mathrm{T}\right)=\frac{i e^{2}}{4 \pi \hbar^{2}} \log \left[\frac{2 \mu-(\omega+\mathrm{i} 2 \Gamma) \hbar}{2 \mu+(\omega+\mathrm{i} 2 \Gamma) \hbar}\right]
$$

A standard 2D graphene sheet is an optically anisotropic material whose diagonal permittivity tensor is written as follows:

$$
\varepsilon_{g}=\left(\begin{array}{ccc}
\varepsilon_{g \|} & 0 & 0 \\
0 & \varepsilon_{g \|} & 0 \\
0 & 0 & \varepsilon_{g \perp}
\end{array}\right)
$$

Here $\varepsilon_{g \perp}=1$ and $\varepsilon_{g \|}=1+i \sigma_{g} / \varepsilon_{0} \omega d, d$ denotes the thickness of the graphene sheet, $\varepsilon_{0}$ signifies vacuum permittivity.

\section{Transfer matrix of graphene-dielectric layered structure}

A photonic crystal is a solid structure with periodically varying dielectric permittivity or inhomogeneity, whose period is comparable with the wavelength of light.

Photonic crystals, thanks to a periodic change in the refractive index, make it possible to obtain allowed and forbidden bands for photon energies, analogous to semiconductor materials in which allowed and forbidden bands are observed for the energy of charge carriers. In practice, this means that if a photon with energy (wavelength/frequency) falls on a photonic crystal, which corresponds to the forbidden band of a given photonic crystal, then it can not propagate in a photonic crystal and is fully reflected back. Conversely, this means that if a photon that has 
energy (wavelength/ frequency) that falls on a photonic crystal corresponds to the allowed band of a given photonic crystal, then it can propagate in a photonic crystal. In other words, the photonic crystal serves as an optical filter that separates electromagnetic waves of various frequencies.

Of interest herein is the 1D photonic structure of dielectric layers, which are separated by the graphene sheets, as shown in Figure 1. The idea of the following consideration is that the graphene sheets have different chemical potentials. During the calculation the incidence of the $\mathrm{p}$-polarized plane wave (TM-wave with the components $\mathrm{E}=$ $\left(E_{x}, 0, E_{z}\right)$ and $\left.H=\left(0, H_{y}, 0\right)\right)$. We essentially focus on the wave propagating along the $\mathrm{Z}$-axis.

It is well known that the wavenumber dispersion of the photonic structure with the same graphene sheet has following form:

$$
k_{z}^{2}=\varepsilon_{d} k_{0}^{2}-\frac{\varepsilon_{d}}{\varepsilon_{g}} k_{t}^{2},
$$

where $\varepsilon_{d}$ is simply the permittivity of the dielectric layer. It can be seen that, depending on the sign of $\varepsilon_{g \|}$, the two modes of the wave propagation are possible: the elliptic modeand the hyperbolic one.Figs. $2 a$ and $2 b$ show the real and imaginary parts of the surface conductivity and permittivity of the graphene. It is clearly seen that for the graphene layer at room temperatures and at the fixed value of the chemical potential $\mu=0.5 \mathrm{eV}$ the real part of the permittivity remains negative in the frequency range higher than $220 \mathrm{Thz}$. The change in the value of the chemical potential $\mu$ shifts the negative region to higher frequencies. In this work we will consider one interesting case when the photonic structure consists of the two types of the periodically repeated graphene sheets with different values of the chemical potential (see Fig. 1).

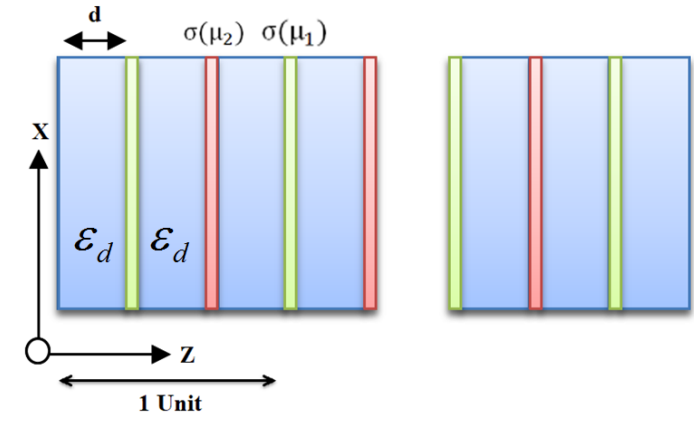

Figure 1 - Layout of the layers of the periodic structure based on the dielectric and the graphene

The transfer-matrix method is widely used in optics to describe how the electromagnetic waves propagate through a stratified medium.

The light reflection due to a single interface between two physically different media is subject to the Fresnel formulas. The situation is not so simple if there are many interfaces because the reflected waves are partially transmitted as well and again partially reflected. Depending on the wavelength and the exact length of the light path, those reflections interfere, either destructively or constructively. The most difficult problem seen in this case is that the overall reflection of a layered structure is a result of an infinite number of reflections at various interfaces.

To avoid such difficulties the transfer matrix method was put forward by exploiting the insight that in view of Maxwell's equations simple continuity conditions can be derived for the electric field at interfaces between two media of distinct physical properties. Thus, the transfer matrix method makes it possible to formally relate the amplitudes of the incident and reflected electromagnetic waves at the input to the layered structure with the amplitudes at the output by means of the matrix $M$ which is obtained by multiplying: the transition matrix $D$ through the interface, and the transmission matrix in the dielectric medium $P_{d}$ :

$$
\begin{gathered}
{\left[\begin{array}{c}
E_{1}^{+} \\
E_{1}^{-}
\end{array}\right]=M\left[\begin{array}{c}
E_{n+1}^{+} \\
E_{n+1}^{-}
\end{array}\right],} \\
M_{T M}=D \cdot P_{d}=\frac{1}{2}\left[\begin{array}{cc}
\left(1+\eta_{T M}+\zeta_{T M}\right) & \left(1-\eta_{T M}-\zeta_{T M}\right) \\
\left(1-\eta_{T M}+\zeta_{T M}\right) & \left(1+\eta_{T M}-\zeta_{T M}\right)
\end{array}\right]\left[\begin{array}{cc}
e^{-i k_{z} d} & 0 \\
0 & e^{i k_{z} d}
\end{array}\right] .
\end{gathered}
$$


Here $\zeta_{T M}=\sigma(\omega, \mu, \Gamma) k_{z} / \varepsilon_{d} \varepsilon_{0} \omega$ stands for the so-called wave impedance, $\sigma(\omega, \mu, \Gamma)$ denotes the conductivity of the graphene determined by the Kubo formula above and $\eta_{T M}=\varepsilon_{d 1} k_{z 2} / \varepsilon_{d 2} k_{z 1}$ is the ratio of the wavenumbers at the adjacent layers of the dielectric media. The latter is equal to 1 of the dielectric medium is the same throughout the metamaterial sample.

Let us consider the simple case when the two graphene sheets are separated by the dielectric layer. Assuming each graphene sheet has the distinct chemical potentials, the transfer matrix of the single unit cell gives rise to the following matrix product:

$$
M=D_{2 \rightarrow 2}\left(\sigma_{1}\right) P(d) D_{2 \rightarrow 2}\left(\sigma_{2}\right) P(d)
$$

To find the dispersion relation we employ the Bloch theory, which advocates that wave is weakened due to passing through each layer of the dielectric according to:

$$
\begin{aligned}
& E_{n+1}^{+}=E_{n}^{+} e^{-\alpha d}, \\
& E_{n+1}^{-}=E_{n}^{-} e^{-\alpha d} .
\end{aligned}
$$

Solving equations (9) - (10) together with relation (6), the following homogeneous linear equation is obtained in the matrix form

$$
\left(\begin{array}{cc}
M_{11}-e^{\alpha d} & M_{12} \\
M_{21} & M_{22}-e^{\alpha d}
\end{array}\right)\left(\begin{array}{l}
E_{n+1}^{+} \\
E_{n+1}^{-}
\end{array}\right)=0,
$$

whose nonzero solution only exists if the determinant of the matrix turns zero

$$
\begin{gathered}
M_{11} M_{22}+e^{2 \alpha d}- \\
-\left(M_{11}+M_{22}\right) e^{\alpha d}-M_{12} M_{21}=0 .
\end{gathered}
$$

Here $\alpha$ is the Bloch wavenumber and $d$ designates the thickness of the dielectric layer located in between the graphene sheets.

Recalling that the condition $M_{11} M_{22}-M_{12} M_{21}=1$ must always be satisfied for a periodic structure, expression (12) is simplified to the following form:

$$
\operatorname{Cosh}(\alpha d)=\left(M_{11}+M_{22}\right) / 2 \text {. }
$$

It is well understood that expression (13) imposes strong restriction on the wavenumbers of electromagnetics waves and is called the dispersion relation.

If $\alpha$ is considered to be a complex number $\alpha=\kappa+i q$ we can easily write the following equation which corresponds to the undamping wave in the periodic structure and strictly determines its bandwidth

$$
\cos (q d)=\cos ^{2}\left(d k_{z}\right)-\frac{i k z \cos \left(d k_{z}\right) \sin \left(d k_{z}\right)}{\varepsilon_{0} \varepsilon_{d} \omega}\left(\sigma_{1}+\sigma_{2}\right)-\sin ^{2}\left(d k_{z}\right)-\frac{k z^{2} \sigma_{1} \sigma_{2} \sin ^{2}\left(d k_{z}\right)}{2 \varepsilon_{0}^{2} \varepsilon_{d}^{2} \omega^{2}}
$$

The dispersion relation (14) describes the law of propagation of the $p$-polarized wave in the periodic photon structure of the two types of the graphene layers with the different values of the chemical potential such that they have different conductivities $\sigma_{1}$ and $\sigma_{2}$. It should be noted that in the subwave regime, at which $k_{z} d \square 1$ and $\beta d \square 1$, expression (14) turns into the dispersion relation obtained on the basis of the effective medium model (5):

$$
k_{z}^{2}=\frac{q^{2}}{4+\frac{2 \mathrm{i}}{d \varepsilon_{0} \varepsilon_{d} \omega}\left(\sigma\left(\mu_{1}\right)+\sigma\left(\mu_{2}\right)\right)}
$$

Taking into account that $k_{z}=\sqrt{\varepsilon_{d} k_{0}^{2}-k_{\perp}^{2}}$ and $k_{\perp}^{2}=k_{x}^{2}+k_{y}^{2}$, the dispersion relation can be rewritten as:

$$
\frac{k_{x}^{2}+k_{y}^{2}}{\varepsilon_{d}}+\frac{q^{2}}{4 \varepsilon_{d}+\frac{2 i}{d \varepsilon_{0} \omega}\left(\sigma\left(\mu_{1}\right)+\sigma\left(\mu_{2}\right)\right)}=1
$$

The analysis of this dispersion relation suggests that the elliptical regime switches to the hyperbolic one when the wavelength varies in a certain range of optical spectra.

Figure 3 shows the dependence of $k_{z}$ on the transverse component of the wave number $k_{\perp}$, 
normalized to $k_{0}$. For numerical calculations the permittivity is taken to be $\varepsilon_{d}=2$ and the width of the dielectric layer is assumed to be $d=80 \mathrm{~nm}$. It is clearly envisaged from this figure that for the fixed values of the chemical potentials $\mu_{1}=0.4 \mathrm{eV}$ and $\mu_{2}=0.8 \mathrm{eV}$ the dependence $k_{z}\left(k_{\perp}\right)$ remains purely elliptic right above the frequency $\omega=1.23 \cdot 10^{15}$
$\mathrm{Hz}$, see Figure $3 \mathrm{~b}$. If the frequency is lowered beyond this critical value the elliptic character of the dispersion goes over to the hyperbolic one, as evidenced by Figure 3a. It has to be mentioned that under ordinary experimental conditions the variation of the chemical potential can be practically realized either by the doping of graphene or by applying an external electric field [10].

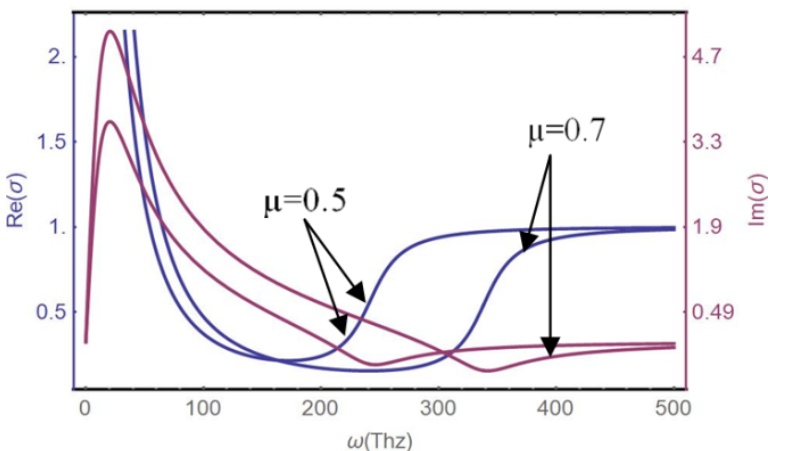

a)

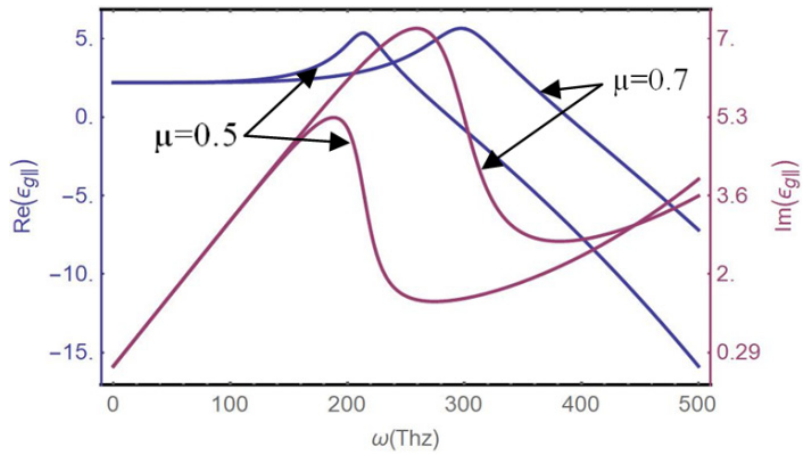

b)

$$
T=300 \mathrm{~K}, \Gamma=0.043 \mathrm{eV}
$$

Figure 2 - The real and imaginary parts of the surface conductivity and the permittivity of the graphene layers

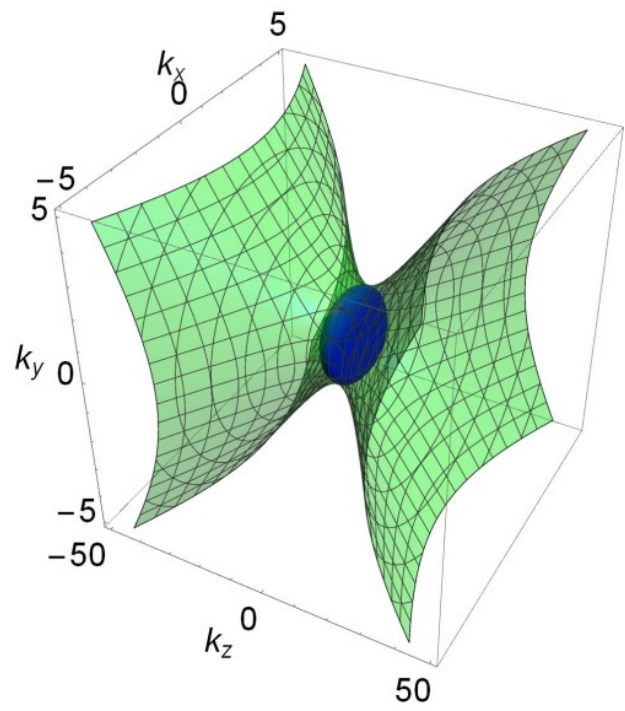

a) $\omega=1.18 \cdot 10^{15} \mathrm{~Hz}$

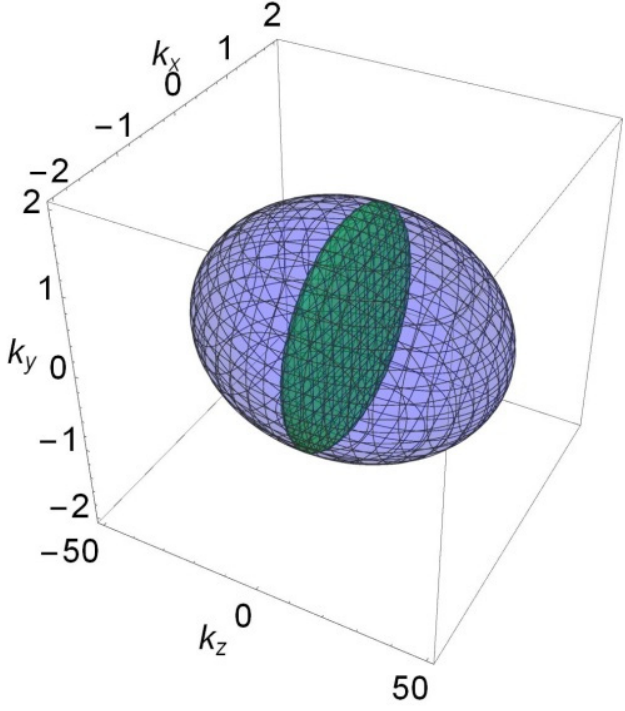

b) $\omega=1.23 \cdot 10^{15} \mathrm{~Hz}$ $T=300 \mathrm{~K}, \Gamma=0.043 \mathrm{eV}, \mu_{1}=0.4, \mu_{2}=0.8$

$T=300 \mathrm{~K}, \Gamma=0.043 \mathrm{eV}, \mu_{1}=0.4, \mu_{2}=0.8$

Figure 3 - The real and imaginary parts of the surface conductivity and the permittivity of the graphene layers 


\section{Spectral characteristics of multilayer graphene-dielectric structures}

A numerical study of the dependence of the transmittance of light on frequency in the periodic photonic structure with the different graphene sheets indicates the existence of the two regions where the transmitted light undertakes jumps. Transmission for a one stack or unit is illustrated in Figure 4 (cf. Fig.1). It is evident from this figure that the value of the chemical potential determines the region of the jump in the transmission spectrum of the light wave. Because the graphene sheets have two different values of the chemical potential $\mu_{1}$ and $\mu_{2}$ the transmission spectrum have a staircase behavior.

Figure 5 shows the transmission and absorbance spectra for different numbers of stacks. The thickness of dielectric layer in this case is $\mathrm{d}=80 \mathrm{~nm}$ and permittivity $\varepsilon=2$. It follows from the figure that increasing the number of stacks leads to a stronger absorptions of light at the second jumped point.

For the dielectric layer of the width $\mathrm{d}=2000 \mathrm{~nm}$ the spectral dependence of transmission gives the

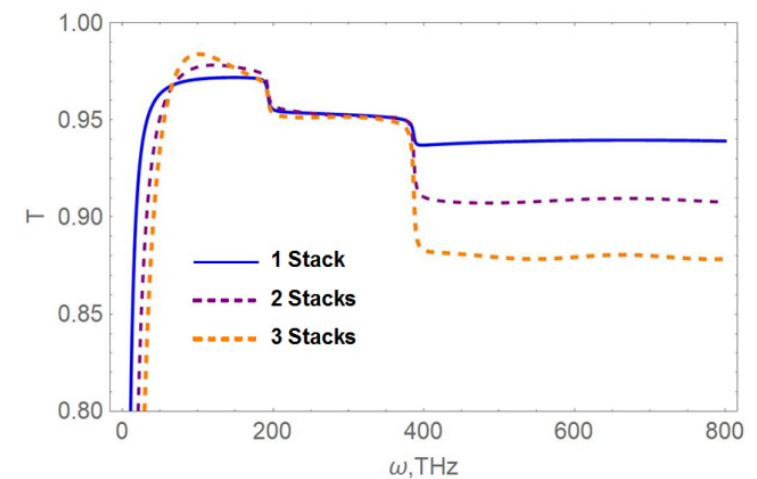

a) similar result which is shown in Figure 6excluding the well pronounced interference pattern.

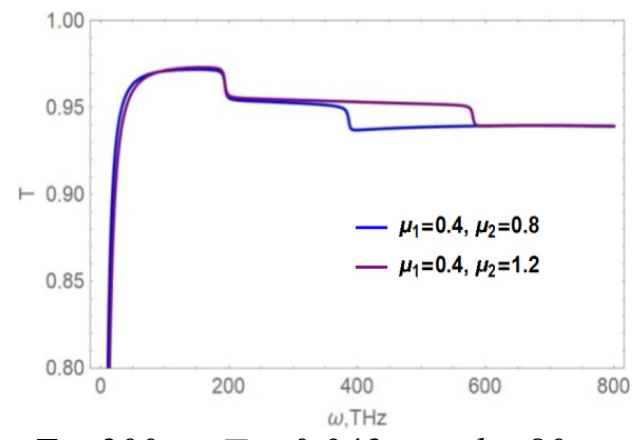

$T=300 \mathrm{~K}, \Gamma=0.043 \mathrm{eV}, d=80 \mathrm{~nm}$

Figure 4 - The transmittance of the photonic structure consisting the two graphene sheets with the different values of the chemical potentials

Figure 7 shows the comparative reflection spectra of the layered structure for several layers at the thicknesses $\mathrm{d}=80 \mathrm{~nm}$ and $\mathrm{d}=2000 \mathrm{~nm}$ of the dielectric layer. In contrast to the transmission and reflection spectra, the spectral dependence of the reflection coefficient does not exhibit a sharp change.

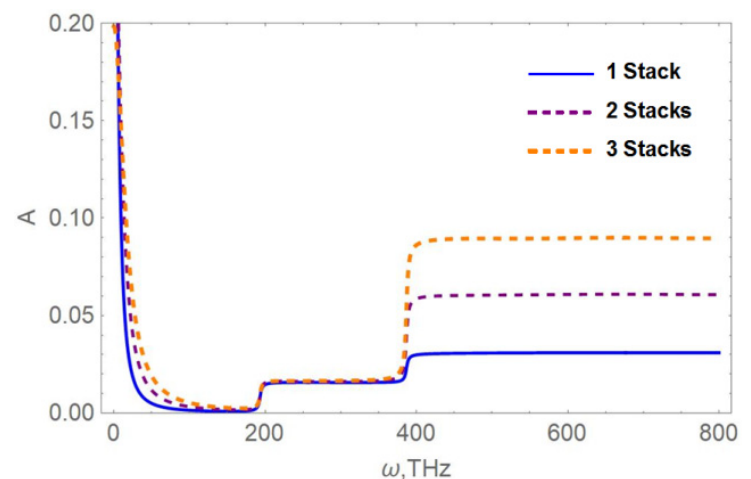

b)

$T=300 \mathrm{~K}, \Gamma=0.043 \mathrm{eV}, \mu_{1}=0.4, \mu_{2}=0.8, d=80 \mathrm{~nm}$

Figure - 5 Transmission (a) and absorbance (b) spectra of the graphene-dielectric layered structure with different number of stacks 


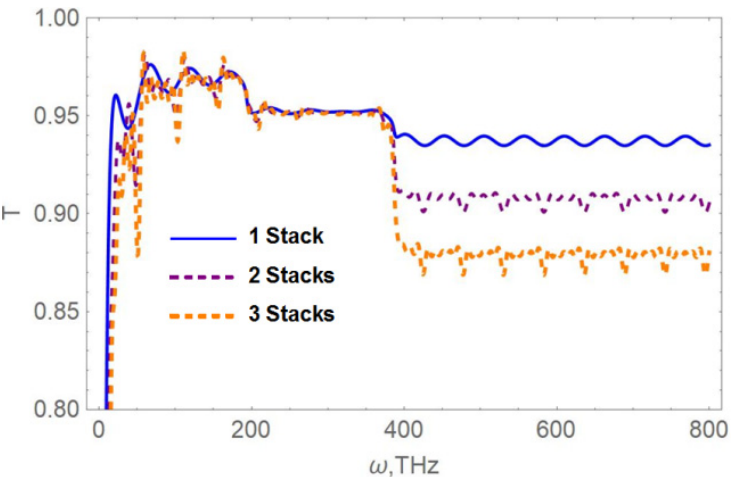

a)

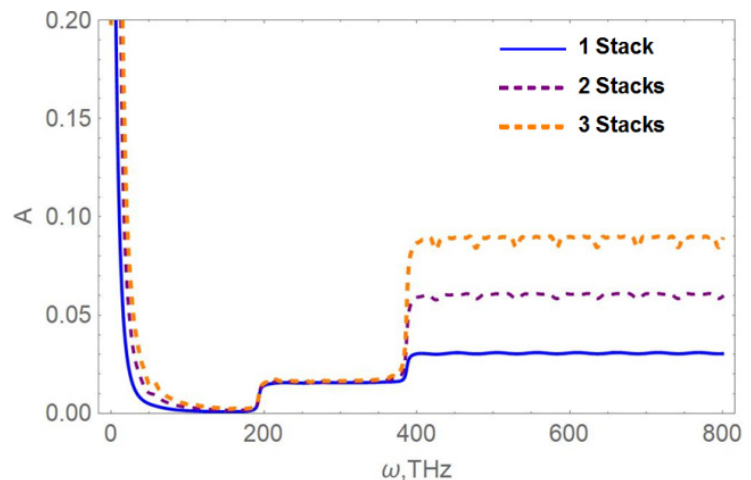

b)

$T=300 \mathrm{~K}, \Gamma=0.043 \mathrm{eV}, \mu_{1}=0.4, \mu_{2}=0.8, d=2000 \mathrm{~nm}$

Figure - 6 Transmission (a) and absorbance (b) spectra of the graphene-dielectric layered structure with different number of stacks

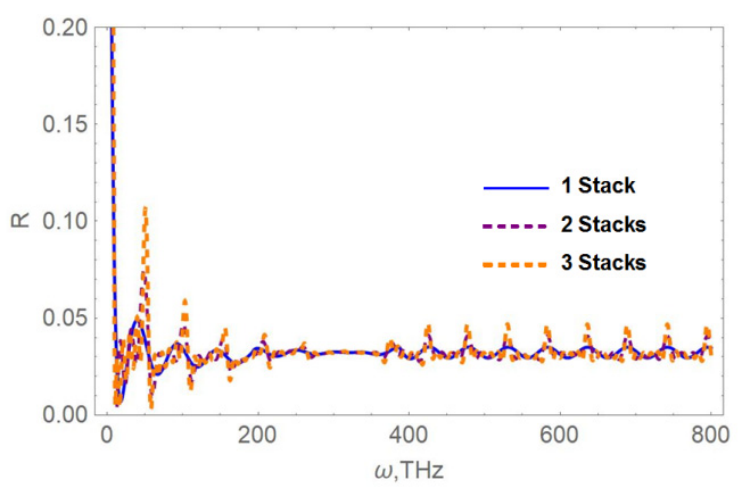

a)

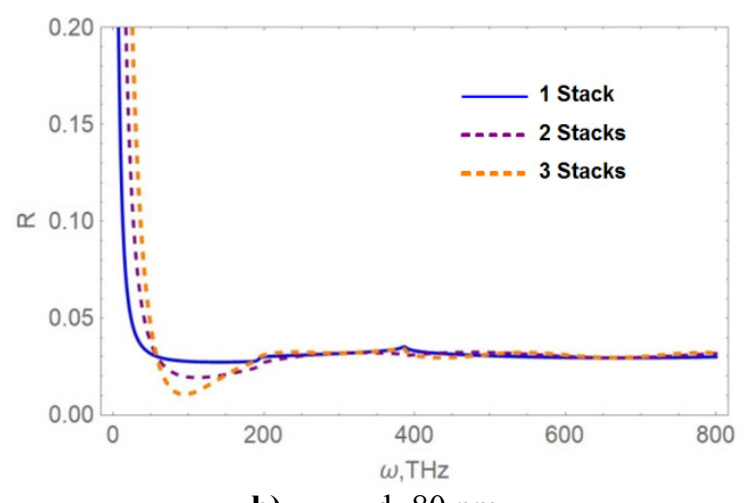

b) $\quad \mathrm{d}=80 \mathrm{~nm}$

$$
T=300 \mathrm{~K}, \Gamma=0.043 \mathrm{eV}, \mu_{1}=0.4, \mu_{2}=0.8
$$

Figure - 7 Reflections spectra of the graphene-dielectric layered structure with different number of stacks

\section{Conclusions}

In this paper we have investigated of the optical characteristics of the photonic structure with the two types of the graphene sheets which are separated by the dielectric medium. The frequency dependent optical conductivity of the graphene sheet is calculated via Kubo formula which takes into account both the interband and the intraband transitions of electrons.

It has been discovered that for the fixed values of the chemical potentials $\mu_{1}=0.4 \mathrm{eV}$ and $\mu_{2}=0.8 \mathrm{eV}$ the dispersion relation $k_{z}\left(k_{\perp}\right)$ is purely elliptic right above the frequency $\omega=1.23 \cdot 10^{15} \mathrm{~Hz}$, whereas for lower frequencies the hyperbolic dispersion persists. The latter regime is known to be responsible for the appearance of the forbidden photon energies which is the characteristic feature of the photonic crystals designed to filter the incoming electromagnetic radiation.

It has been found that for a dielectric layers of width $\mathrm{d}=2000 \mathrm{~nm}$ the interference pattern occur in contrast to the case of $\mathrm{d}=80 \mathrm{~nm}$. This can be evidently understood by recalling the fact that the frequencies and the wavelengths of interest herein all correspond to the optical spectrum.

It has to be inferred that when the frequency of the incoming electromagnetic wave lies in the frequency range,strictly corresponding to the forbidden bands of the photonic crystal, the wave 
itself is totally reflected, whereas the transmitted wave at the output of the sample reaches its minimal intensity.In view of the results obtained, it is straightforward to conclude that owing to its unusual optical properties the graphene proves to be a more promising material for fabricating new generation of the hyperbolic metamaterials than conventional metallic structures. The latter speculation entirely stems from the experimental insight that the light passing through a layered structure is willingly manipulated by an appropriate variation of the chemical potential of the graphene sheets.

\section{Acknowledgements}

The authors acknowledge the financial support from the state grant number 3120/GF4, funded by the Ministry of Education and Science of the Republic of Kazakhstan.

\section{References}

1 Istrate E., Sargent E. H. Photonic crystal heterostructures and interfaces // Rev.Mod.Phys. - 2006. - Vol. 78. - P. 455-482.

2 Shelby R.A., Smith D.R., Schultz S. Experimental verification of a negative index of refraction // Science. - 2001. - Vol. 292. - P. 77-79.

3 Poddubny A., Iorsh I., Belov P., Kivshar Yu. Hyperbolic metamaterials // Nat. Photon. - 2013. Vol. 7. - P. 958-967.

4 Kabashin A.V. Evans P., Pastkovsky S., Hendren W., Wurtz G.A., Atkinson R., Pollard R., Podolskiy V.A., Zayats A.V. Plasmonic nanorod metamaterials for biosensing // Nature materials. - 2009. -Vol. 8. - P. 867-871.

5 Wurtz G.A., Pollard R., Hendren W., Wiederrecht G.P., Gosztola D.J., Podolskiy V.A., Zayats A.V. Designed ultrafast optical nonlinearity in a plasmonicnanorodmetamaterial enhanced by nonlocality// Nature nanotechnology. - 2011. - Vol. 6. P.107-111.

6 Geim A. K.,Novoselov K. S. Two-dimensional gas of massless Dirac fermions in grapheme// Nature. 2005. - Vol. 438. - P. 197-200.
7 Othman A.K., Guclu C., Capolino F. Graphenedielectric composite metamaterials: evolution from elliptic to hyperbolic wavevector dispersion and the transverse epsilon-near zero condition // Journal of Nanophotonics. - 2013.-Vol.7. -P.073089(1-15).

8 Zhan T., Shi Xi. Dai. Yu. Transfer matrix method for optics in graphene layers // Journal of Physics: Condensed matter. -2013. -Vo.45.-No.21.

9 Novoselov K. S. Two-dimensional gas of massless Dirac fermions in graphene // Nature. - 2005. Vol. 438 (7065). - P.197-200.

10 Kim S.J., Park S.J., Kim H.Y. Characterization of chemical doping of graphene by in-situ Raman spectroscopy // Appl.Phys.Lett. -2016. -Vol.108. No.203111.

11 Bao Q., Loh K. P. Graphene photonics, Plasmonics, and broadband optoelectronic devices// ACS Nano - 2012. - Vol. 6. - P. 3677-3694.

12 Long Ju, BaisongGeng, Jason Horng, CaglarGirit, Michael Martin, Zhao Hao, and Hans A. Bechtel. Graphene plasmonics for tunable terahertz metamaterials. // Nature nanotechnology - 2011. - Vol. $6(10) .-$ P. 630-634. 